\title{
The World of Embryo Morphokinetics of Time-Lapse Systems
}

In the last 6 years, several time-lapse ( $T L)$ image systems have been introduced with the aim of improving the parameters of success in IVF/ICSI. The continuous observation in the laboratory of images of embryonic development combined with reduced handling of embryos is alluring for embryologists. In addition, the ability to review the morphokinetic aspects at any time creates a favorable basis for decreasing the variability associated with individual analyses.

Thus, from the classical TL parameters (cleavage stage: $24-27 \mathrm{~h}$, 4-cell embryos: $49 \pm 1.3$ hours, and 8-cell embryos: $64.8 \pm 1.8$ hours), new TL parameters were created (extrusion time of the 2nd polar body, disappearance time of the pronuclei, and duration of blastulation) with the ultimate goal of improving the accuracy of the embryo selection process (Cyray et al., 2014). TL systems can be installed in a common everyday incubator or may be present in new specialized incubators. Therefore, the success of TL systems in achieving IVF/ICSI should be analyzed both in terms of the new embryo morphokinetics and the efficiency of the incubator used for embryo culture. The basic strategy is to increase the specificity of the morphology variable in the identification of the ideal embryo quality (embryo health), thus leading to a higher rate of implantation, pregnancy and newborns (healthy pregnancy and fetus).

Are TL systems more efficient in embryo selection than classical morphological analysis? If that was the case, ART laboratories would almost be obliged to purchase TL systems, which are currently more costly.

On the other hand, randomized studies in patients are always more effective for determining the clinical efficacy parameters of a methodology of IVF/ICSI, such as the evolution of pregnancy and/or newborns born to patients undergoing a particular intervention (Armstrong et al., 2015). Currently, only one published randomized patient study has been reported (Rubio et al., 2014). These authors used a prospective and random analysis of a total of 856 patients (TL group $=444$; control group = 412). In the TL group, a TL designated incubator and TL-specific morphokinetics were used, whereas in the control group, the classical morphological parameters and a traditional incubator were used. The implantation rates and the evolution of pregnancy were significantly more favorable using the TL system. However, these factors were only significant in patients who underwent blastocyst embryo transfers (day 5), whereas for patients receiving cleavage stage embryo transfers on day 3 , the success rates of IVF/ICSI were not statistically significant. At this point, it is worth recalling that traditional incubators do not work with $5 \% \mathrm{O} 2$, the growth condition that is now accepted as ideal for efficient culturing of cleaved embryos until the blastocyst stage. Additionally, the study did not use a standardized day for embryo transfer, which resulted in a number of patients in which the determination of the best day (day 3 or 5 ) for evaluation of the culture system by the time-lapse system was less than ideal. In addition, half of the patients received embryos from egg donation programs, which limited the use of information for populations actually defined as infertile (poor prognosis).

To date, evidence-based medicine has not shown that time-lapse systems have any real advantages over classical morphological analyses. We will therefore have to wait for new prospective and randomized studies to compare TL systems with traditional methods before this methodology is definitely adopted. If, over time, the high cost of these technologies significantly decreases and their efficacy is at least similar to that of traditional embryo culture, the world of morphokinetics of TL systems cannot be completely ignored.

José Gonçalves Franco Jr

President of SBRA - Brazilian Society of Assisted Reproduction

Scientific Director of Center for Human Reproduction Prof. Franco Jr, Ribeirao Preto, SP, Brazil

\section{REFERENCES}

Armstrong S, Vail A, Mastenbroek S, Jordan V, Farquhar C. Time-lapse in the IVF-lab: how should we assess potential benefit? Hum. Reprod. 2015; 30:3-8.

Cyray HN, Campbell A, Agerholm IE, Aguillar J, Chamayou S, Esbert M, Sayed S. Proposed guidelines on the nomenclature and annotation of dynamic human embryo monitoring by a time-lapse user group. Hum. Reprod. 2014;29:2650-60.

Rubio I, Galán A, Larreateguí Z, Ayerd F, Beliver J, Herrero J, Meseguer M. Clinical validation of embryo culture and selection by morphokinetic analysis: a randomized, controlled trial of the Embryoscope. Fertil. Steril. 2014;102:1287-94. 